\title{
Comparison of serum exosome isolation methods on co- precipitated free microRNAs
}

\author{
Yirui Cheng ${ }^{1}$, Xiangyun Qu ${ }^{2}$, Zhaonan Dong ${ }^{2}$, Qingyu Zeng ${ }^{1}$, Xueqing Ma ${ }^{2}$, Yunli Jia ${ }^{2}$, Ruochen Li ${ }^{2}$, Xiaoxu Jiang \\ ${ }^{2}$, Cecilia Williams ${ }^{3}$, Tao Wang ${ }^{2}$, Weiliang Xia ${ }^{\text {Corresp. } 1}$ \\ ${ }^{1}$ School of Biomedical Engineering and Med-X Research Institute, Shanghai Jiao Tong University, Shanghai, China \\ Jiangsu Cancer Molecular Diagnostics Engineering Research Center, Suzhou MicroDiag Biomedicine Co., Ltd, Suzhou, China \\ 3 Department of Protein Science, KTH Royal Institute of Technology, Science for Life Laboratory, Solna, Sweden \\ Corresponding Author: Weiliang Xia \\ Email address: wlxia@sjtu.edu.cn
}

Background. Exosomes are nano-sized extracellular vesicles containing different biomolecules such as proteins and microRNAs (miRNAs) that mediate intercellular communication. Recently, numerous studies have reported the important functions of exosomal miRNAs in disease development and the potential clinical application as diagnostic biomarkers. Up to now, the most commonly used methods to extract exosomes are ultracentrifugation (UC) and precipitation-based commercial kit (e.g., ExoQuick). Generally, both UC and ExoQuick method could co-isolate contaminating proteins along with exosomes, with the UC method yielding even purer exosomes than ExoQuick. However, the comparison of these two methods on co-precipitated free miRNAs is still unknown.

Methods. In this study, we isolated exosomes from the human serum with exogenously added celmiR-39 by UC and ExoQuick and compared the proportion of cel-miR-39 co-precipitated with exosomes extracted by these two methods.

Results. Using exogenous cel-miR-39 as free miRNAs in serum, we concluded that ExoQuick co-isolates a small proportion of free miRNAs while UC hardly precipitates any free miRNAs. We also found that incubation at $37^{\circ} \mathrm{C}$ for $1 \mathrm{~h}$ could decrease the proportion of free miRNAs, and exosomal miRNAs like miR-126 and miR-152 also decreased when RNase A was used. In conclusion, our findings provide essential information about the details of serum exosome isolation methods for further research on exosomal miRNAs. 


\section{Comparison of Serum Exosome Isolation Methods on 2 Co-precipitated Free microRNAs}

3

4

5

6

7

Yirui Cheng ${ }^{1}$, Xiangyun $\mathrm{Qu}^{2}$, Zhaonan Dong ${ }^{2}$, Qingyu Zeng ${ }^{1}$, Xueqing $\mathrm{Ma}^{2}$, Yunli $\mathrm{Jia}^{2}$, Ruochen $\mathrm{Li}^{2}$, Xiaoxu Jiang ${ }^{2}$, Cecilia Williams ${ }^{3}$, Tao Wang ${ }^{2}$, Weiliang Xia ${ }^{1}$

${ }^{1}$ School of Biomedical Engineering and Med-X Research Institute, Shanghai Jiao Tong University, Shanghai 200030, PR China

${ }^{2}$ Jiangsu Cancer Molecular Diagnostics Engineering Research Center, Suzhou MicroDiag Biomedicine Co., Ltd, Suzhou 215028, PR China

${ }^{3}$ Department of Protein Science, KTH Royal Institute of Technology, Science for Life Laboratory, Solna, Sweden.

Corresponding Author:

Weiliang $\mathrm{Xia}^{1}$

No.1954, Huashan Road, Shanghai, 200030, PR China

Email address: wlxia@sjtu.edu.cn

\section{Abstract}

Background. Exosomes are nano-sized extracellular vesicles containing different biomolecules such as proteins and microRNAs (miRNAs) that mediate intercellular communication. Recently, numerous studies have reported the important functions of exosomal miRNAs in disease development and the potential clinical application as diagnostic biomarkers. Up to now, the most commonly used methods to extract exosomes are ultracentrifugation (UC) and precipitationbased commercial kit (e.g., ExoQuick). Generally, both UC and ExoQuick method could coisolate contaminating proteins along with exosomes, with the UC method yielding even purer exosomes than ExoQuick. However, the comparison of these two methods on co-precipitated free miRNAs is still unknown.

Methods. In this study, we isolated exosomes from the human serum with exogenously added cel-miR-39 by UC and ExoQuick and compared the proportion of cel-miR-39 co-precipitated with exosomes extracted by these two methods.

Results. Using exogenous cel-miR-39 as free miRNAs in serum, we concluded that ExoQuick co-isolates a small proportion of free miRNAs while UC hardly precipitates any free miRNAs.

We also found that incubation at $37^{\circ} \mathrm{C}$ for $1 \mathrm{~h}$ could decrease the proportion of free miRNAs, and exosomal miRNAs like miR-126 and miR-152 also decreased when RNase A was used. In conclusion, our findings provide essential information about the details of serum exosome isolation methods for further research on exosomal miRNAs.

Key words: exosome, isolation method, ultracentrifugation, ExtraPEG, microRNAs, serum 
41

42

43

44

45

46

47

48

49

50

51

52

53

54

55

56

57

58

59

60

61

62

63

64

65

66

67

68

69

70

71

72

73

74

75

76

77

78

79

\section{Introduction}

Exosomes are small secreted extracellular vesicles of 30-200 $\mathrm{nm}$ in diameter with the same structure as cell membrane (Pegtel \& Gould 2019; Thery et al. 2002; Tkach \& Thery 2016). They convey intercellular communications by delivery of biomolecules and affect multiple physiological processes under normal or diseased conditions (He et al. 2018; Meckes et al. 2010; Valadi et al. 2007). They originate from multivesicular bodies (MVBs) which contain many small vesicles called intraluminal endosomal vesicles (ILVs) and the ILVs become exosomes when the MVBs fuse with the plasma membrane, releasing the ILVs into the extracellular space (Colombo et al. 2014). Moreover, exosomes have been found in diverse biological fluids, such as serum, urine, saliva and breast milk (Thery et al. 2006).

MicroRNAs (miRNAs), a class of 19-23 nt non-coding RNAs are known as a mediator of post-transcriptional regulation, which can negatively regulate the expression of target mRNAs (Bartel 2004). While the majority of miRNAs are located within the cell, recently, significant number of miRNAs have been found in extracellular environment, including various biological fluids and cell culture media, commonly known as circulating miRNAs or extracellular miRNAs (Turchinovich et al. 2011). Recently, an increasing number of extracellular miRNAs have been detected in exosomes isolated from biological fluids and cell culture media (Valadi et al. 2007). Moreover, some exosomal miRNAs have been shown to regulate disease development and reported as biomarkers of different types of cancers, cardiovascular disease and brain injury (Grimolizzi et al. 2017; Lugli et al. 2015; Zhang et al. 2017). In addition to exosomal miRNAs, there are still some circulating miRNAs free in the serum (Mitchell et al. 2008). It remains unclear whether these free miRNAs can be co-precipitated with exosomes and how to remove these free miRNAs from exosome pellets if so.

The most commonly used methods to extract exosomes are ultracentrifugation (UC) and commercially available kits, like ExoQuick from Systems Biosciences, exploiting sedimentation, with pros and cons for each method (Alvarez et al. 2012; Umezu et al. 2013). Generally, UC is the most reliable "gold standard" method but time-consuming; and precipitation methods such as ExoQuick can obtain higher yields of exosomes but co-precipitate more impure proteins at the same time (Shao et al. 2016). However, if and to what extent these two exosome isolation methods can co-isolate serum free miRNAs remain to be investigated.

In this study, regarding exogenous cel-miR-39 as free miRNAs in serum, we concluded that ExoQuick co-isolates a small proportion of free miRNAs while UC hardly precipitates any free miRNAs. Further, we found that incubation at $37^{\circ} \mathrm{C}$ for $1 \mathrm{~h}$ can decrease the proportion of free miRNAs in exosome pellets and exosomal miRNAs like miR-126 and miR-152 (Grimolizzi et al. 2017; Lugli et al. 2015; Ong et al. 2014; Zhang et al. 2017) also decreased when RNase A was used. In conclusion, our findings provide essential information on the details of serum exosome isolation methods for further studies on exosomal miRNAs.

\section{Materials \& Methods}


80

81

82

83

84

85

86

87

88

89

90

91

92

93

94

95

96

97

98

99

100

101

102

103

104

105

106

107

108

109

110

111

112

113

114

115

116

117

118

\section{Sample collection}

Human blood samples were collected into serum collection tubes from the Shanghai Chest Hospital. Blood samples were centrifuged at $3000 \mathrm{rpm}$ at $4{ }^{\circ} \mathrm{C}$ for $10 \mathrm{~min}$ for serum collection, followed by an additional centrifugation step at $3000 \mathrm{~g}$ for $15 \mathrm{~min}$. The supernatants of different samples were mixed and then equally divided into several portions $(500 \mu \mathrm{L})$ for subsequent experiments.

This study was approved by the Ethical Committee of the School of Biomedical Engineering, Shanghai Jiao Tong University (Registration No. 2019045) and carried out in accordance with the Declaration of Helsinki. All participants provided written informed consent.

\section{Exogenous miRNA addition}

The serum $(500 \mu \mathrm{L})$ were added with $5 \mu \mathrm{L} 7 \times 10^{9}$ copies $/ \mu \mathrm{L}$ cel-miR-39 or $5 \mu \mathrm{L} 7 \times 10^{6}$ copies/ $\mu \mathrm{L}$ cel-miR-39 or $5 \mu \mathrm{LddH} \mathrm{H}_{2} \mathrm{O}$. The sequence of cel-miR-39 is: forward 5'UCACCGGGUGUAAAUCAGCUUG-3'.

\section{Exosome isolation}

ExoQuick ${ }^{T M}$ Kit

Exosomes from $500 \mu \mathrm{L}$ serum were isolated by ExoQuick ${ }^{\mathrm{TM}}$ kit (System Biosciences Inc., CA, USA) according to the manufacturer's recommendations. Briefly, $120 \mu \mathrm{L}$ EXOQ5A-1 was added to serum and the mixture was incubated at $4{ }^{\circ} \mathrm{C}$ for $30 \mathrm{~min}$. After incubation, the mixture was centrifuged at $13000 \mathrm{rpm}$ for $2 \mathrm{~min}$. The supernatant was removed and the exosome pellet was used for subsequent experiments.

\section{Ultracentrifugation (UC)}

Exosomes from $500 \mu \mathrm{L}$ serum were also isolated by UC method. Briefly, the serum was transferred into $12.5 \mathrm{~mL}$ ultracentrifuge tube (Beckman). The tube then was filled with PBS (HyClone, SH30256.01) followed by ultracentrifugation twice for $70 \mathrm{~min}$ at $100,000 \mathrm{~g}, 4{ }^{\circ} \mathrm{C}$ in an SW 40Ti swinging-bucket rotor (Beckman). The supernatant was discarded and the exosome pellet was used for subsequent experiments.

\section{Exosome identification}

Transmission electron microscopy (TEM)

The exosome pellet was resuspended in a small volume of PBS. According to Thery et al (Thery et al. 2006), the exosomes ( $5 \mu \mathrm{L}$ ) was dripped onto a copper grid (Zhongjingkeyi, CHN, BZ110223b) firstly. After one minute, the droplet was sucked out using the air-laid paper. And then, the $2 \%$ uranyl acetate (Merck, 1005) solution $(5 \mu \mathrm{L})$ was dripped onto the same copper grid for negative-staining and sucked out again one minute later.

Nanoparticle tracking analysis (NTA)

The size distribution of the exosome pellet was measured by ZetaView (Particle Metrix). The pellet was resuspended in a proper volume of PBS to achieve the optimal detectable 
119 concentration (about $10^{7}$ particles per $\mathrm{mL}$ ) of its corresponding software (ZetaView 8.03.04.01).

120 For each measurement, 3-5 mL of the diluted sample was injected into the machine.

121 Western blot

122 Western blotting was performed according to the manufacturer's instruction manual. Briefly, 123 each pellet $(30 \mu \mathrm{g})$ was mixed with loading buffer $(5 \mathrm{x})$ and heated at $95{ }^{\circ} \mathrm{C}$ for $5 \mathrm{~min}$. Proteins were loaded on 10\% sodium dodecyl sulfate polyacrylamide gel (SDS-PAGE; EpiZyme, PG112) and separated at constant $55 \mathrm{~V}$ for $30 \mathrm{~min}$ first and then $120 \mathrm{~V}$ until the loading buffer running out of the SDS-PAGE. Then, proteins were transferred to a nitrocellulose membrane (GE Healthcare, 10600002) at constant $300 \mathrm{~mA}$ for $1.5 \mathrm{~h}$. Next, the membrane was blocked with 5\% nonfat milk powder suspended in tris-buffered saline and tween 20 (TBST) for $1 \mathrm{~h}$ at room temperature. The blots were probed with TSG101 (Abcam, 133586) and subsequently probed with horseradish peroxidase conjugated secondary anti-rabbit antibodies (Jackson). Finally, the blots were visualized using the enhanced chemiluminescent (ECL; Thermo, 1856136) and chemiluminescent imaging system (Tanon, 5200).

133

134

\section{RNase $A$ and RNase Inhibitor treatment}

136

The exosome pellet was suspended in $500 \mu \mathrm{L}$ PBS and a determined volume of RNase A (Thermo Scientific, MAN0012003) was added in the RNase A treatment groups to reach a final concentration of $10 \mu \mathrm{g} / \mathrm{mL}, 20 \mu \mathrm{g} / \mathrm{mL}$ or $30 \mu \mathrm{g} / \mathrm{mL}$. And RNase inhibitor (Thermo Scientific, EO0381) was added in the RNase inhibitor treatment groups to reach a final concentration of 1 $\mathrm{U} / \mu \mathrm{L}$. Then the mixture was incubated at $37^{\circ} \mathrm{C}$ for $1 \mathrm{~h}$.

140

\section{RNA isolation}

Exosomal RNA was extracted by miRNeasy Mini Kit (Qiagen, 217004) according to the manufacturer's recommendations. The final elution volume was $35 \mu \mathrm{L}$.

\section{Reverse Transcription Polymerase Chain Reaction (RT-PCR)}

The cDNA of cel-miR-39, miR-126 and miR-152 were synthesized with the Revert Aid First Strand cDNA Synthesis Kit (Thermo Scientific, K1622). Briefly, $5 \mu \mathrm{L}$ total RNA from each sample were mixed with $5 \mu \mathrm{L}$ of RT-Primer, $2 \mu \mathrm{L}$ of $10 \times \mathrm{T} 4$ Buffer, $2 \mu \mathrm{L}$ of $10 \mathrm{mM}$ dNTP Mix, 1 $\mu \mathrm{L}$ of RNase inhibitor, $1 \mu \mathrm{L}$ of Transcriptase, $0.5 \mu \mathrm{L}$ of T4 Polynucleotide Kinase, $0.25 \mu \mathrm{L}$ of T4 Kinase, and $3.25 \mu \mathrm{L}$ of nuclease-free water. Then the $20 \mu \mathrm{L}$ of mixture was incubated at $16{ }^{\circ} \mathrm{C}$ for $30 \mathrm{~min}, 42{ }^{\circ} \mathrm{C}$ for $30 \mathrm{~min}, 85{ }^{\circ} \mathrm{C}$ for $5 \mathrm{~min}$ followed by cooling at $4{ }^{\circ} \mathrm{C}$ in the PCR instrument (LongGene, A100).

Note that $5 \mu \mathrm{L}$ of $35 \mu \mathrm{L}$ extracted RNA were used in RT-PCR, so if all the exogenous celmiR-39 had been co-isolated with exosomes, the current concentration of cel-miR-39 in this procedure would be $10^{9}$ copies $/ \mu \mathrm{L}$ or $10^{6}$ copies $/ \mu \mathrm{L}$. Therefore, $10^{9}$ copies $/ \mu \mathrm{L}$ or $10^{6}$ copies $/ \mu \mathrm{L}$ cel-miR-39 were used as a standard control.

Here, stemloop RT primers were used for cDNA synthesis. The sequence of miR-126 is: 5'UCGUACCGUGAGUAAUAAUGCG-3'. The RT primer of miR-126 is: 5'- 
159

160

161

162

163

164

165

166

167

168

169

170

171

172

173

174

175

176

177

178

179

180

181

182

183

184

185

186

187

188

189

190

191

192

193

194

195

196

197

198

GATGAGGAGTGTCGTGGAGTCGGCAATTTCCTCATCACGCATTA-3'. The sequence of miR-152 is: 5'-AGGUUCUGUGAUACACUCCGACU-3'. The RT primer of miR-152 is: 5'GATGAGGAGTGTCGTGGAGTCGGCAATTTCCTCATCAAGTCGGAG-3'. The RT primer of cel-miR-39 is: 5'- GATGAGGAGTGTCGTGGAGTCGGCAATTTCCTCATCCAAGCTG 3 .

\section{Quantitative real-time PCR ( $q R T-P C R)$}

To validate the quantity of cel-miR-39, miR-126 and miR-152 in the RNA samples, qPCR was performed using Fluorescence Polymerase Chain Reaction (PCR) Detection Kit for the Analysis of Human miRNA Gene Expression (Jiangsu MicroDiag Biomedicine Technology Co. Ltd., China) according to the manufacturer's recommendations. Briefly, the synthesized cDNA of celmiR-39, miR-126 and miR-152 from each sample were mixed with $10 \times$ Buffer, $25 \mathrm{mM} \mathrm{MgCl}$, $25 \mathrm{mM}$ dNTP Mix, F-primer, R-primer, miRNA-specific probe, HS Tag, UDG and ddH2O. Meanwhile, $10^{2}-10^{7}$ copies of cel-miR-39, miR-126 and miR-152 were amplificated in this procedure to get a curve for calculating miRNA copy numbers in each sample.

The PCR reaction mixture was incubated at $37{ }^{\circ} \mathrm{C}$ for $5 \mathrm{~min}$, and then $94{ }^{\circ} \mathrm{C}$ for $5 \mathrm{~min}$, followed by 50 cycles of $94{ }^{\circ} \mathrm{C}$ for $15 \mathrm{sec}, 60{ }^{\circ} \mathrm{C}$ for $60 \mathrm{sec}$, and cooling at $50{ }^{\circ} \mathrm{C}$ for $30 \mathrm{sec}$, which was performed using a real-time fluorescent quantitative PCR instrument (Roche, LightCycler 480). Here, the miRNA-specific probes were used. The F-primer of miR-126 is: 5'CTCCGTCAGGGTCGTACCGTGAGTAA-3'. The R-primer of miR-126 is: 5'CTCAAGTGTCGTGGAGTCGGC-3'. The specific probe of miR-126 is: 5'-FAMTTTCCTCATCACGCATTA-MGB-3'. The F-primer of miR-152 is: 5'CTCCGTCAGGGAGGTTCTGTGATACA-3'. The R-primer of miR-152 is: 5'CTCAAGTGTCGTGGAGTCGGC-3'. The specific probe of miR-152 is: 5'-FAMTTTCCTCATCAAGTCGGAG-MGB-3'. The F-primer of cel-miR-39 is: 5'CGTATGAGCGTCACCGGGTGTAAATCA-3'. The R-primer of miR-152 is: 5'CTCAAGTGTCGTGGAGTCGGCAA-3'. The specific probe of miR-152 is: 5'-FAMTTTCCTCATCCAAGCTG-MGB-3'.

\section{Statistical analysis}

Each experiment was repeated at least three times. Data were analyzed and all statistical graphs were generated by GraphPad Prism 6.0 (GraphPad Software Inc, La Jolla, CA, USA). Differences between two groups were analyzed using one-way or two-way ANOVA and the probability value below 0.05 was considered significant.

\section{Results}

\section{Workflow of this research and the identification of exosomes}

The experimental procedure of this research is summarized (Fig. 1), with details given in subsequent sections. First, $5 \mu \mathrm{L} 7 \times 10^{9}$ copies $/ \mu \mathrm{L}$ or $7 \times 10^{6}$ copies $/ \mu \mathrm{L}$ exogenous cel-miR-39 was added in $500 \mu \mathrm{L}$ human serum while same volume of $\mathrm{ddH}_{2} \mathrm{O}$ was added in another group as 
199

200

201

202

203

204

205

206

207

208

209

210

211

212

213

214

215

216

217

218

219

220

221

222

223

224

225

226

227

228

229

230

231

232

233

234

235

236

237

238

blank control (step I). Next, the exosomes were isolated by ExoQuick or UC method (step II) and characterized using TEM, NTA and western blot (Fig. 2(a-f)). TEM showed exosomes' "cupshaped" morphology and co-isolated impure proteins in both methods (Fig. 2(a, d)). NTA analysis showed exosomes' similar particle size distribution mostly between 30-200 nm (Fig. 2(b, d)), however, ExoQuick-isolated exosomes tended to be a little smaller than those from UC, which may be caused by more co-precipitated impure proteins from ExoQuick method. Furthermore, exosomes from both methods obtained the exosome-associated protein marker, CD63, TSG101, CD9 (Fig. 2(e, f)) (Thery et al. 2018). Following, to clear up the co-isolation free miRNA in exosome pellets, $10 \mu \mathrm{g} / \mathrm{mL}, 20 \mu \mathrm{g} / \mathrm{mL}$ or $30 \mu \mathrm{g} / \mathrm{mL}$ RNase A were added in RNase A group and incubated with exosomes at $37{ }^{\circ} \mathrm{C}$ for $1 \mathrm{~h}$. Moreover, $1 \mathrm{U} / \mu \mathrm{L}$ RNase inhibitor were used to eliminate the effect of environmental RNase; the control group was a blank control with no enzyme addition, which was also placed at $4{ }^{\circ} \mathrm{C}$ for $1 \mathrm{~h}$ (step III). Then, total RNA including miRNA of exosomes was extracted by QIAGEN miRNeasy Mini Kit and cel-miR-39, miR-126 or miR-152 in exosome samples were detected using RT-PCR followed by qPCR. $5 \mu \mathrm{L} 10^{9}$ copies $/ \mu \mathrm{L}$ or $10^{6}$ copies $/ \mu \mathrm{L}$ cel-miR-39 was used as standard control in PCR procedure (step IV, V and VI).

\section{ExoQuick co-isolates a small proportion of free miRNAs}

As described in Figure 1, higher concentration $\left(7 \times 10^{9}\right.$ copies $\left./ \mu \mathrm{L}\right)$ of cel-miR-39 was added in $500 \mu \mathrm{L}$ serum and then exosomes were extracted by ExoQuick method. The ratio of cel-miR-39 in exosome samples to standard cel-miR-39 sample $\left(10^{9}\right.$ copies $\left./ \mu \mathrm{L}\right)$ showed that about $2 \%$ exosome-independent cel-miR-39 were co-isolated with exosomes extracted by ExoQuick method (Fig. 3(a)). To measure the effect of RNase A to remove exosome-independent miRNAs, $10 \mu \mathrm{g} / \mathrm{mL}, 20 \mu \mathrm{g} / \mathrm{mL}, 30 \mu \mathrm{g} / \mathrm{mL}$ RNase A and $1 \mathrm{U} / \mu \mathrm{L}$ RNase inhibitor were respectively added in the same exosome samples, and the results of PCR showed that RNase A can decrease the quantity of co-isolated cel-miR-39 in exosomes but there are no significance difference between different concentrations of RNase A and RNase inhibitor groups (Fig. 3(a)). Moreover, RNase A decreased the quantity of exosomal miR-126 and miR-152 at the same time compared with control and RNase inhibitor groups (Fig. 3(b)). Interestingly, RNase inhibitor reduced the amount of exosomal miR-126 but not reduced miR-152 (Fig. 3(b)).

To verify if the co-isolation is due to the high concentration of cel-miR-39, low concentration $\left(7 \times 10^{6}\right.$ copies $\left./ \mu \mathrm{L}\right)$ of miR-39 were exogenously added in serum. Similar to the results of high concentration cel-miR-39 experiment, nearly $2 \%$ cel-miR-39 were co-isolated with exosomes and RNase A decreased the amount of exosomal miR-126 and miR-152 (Fig. 3(c-d)). And RNase inhibitor reduced the amount of exosomal miR-126, not the miR-152 in low concentration cel-miR-39 experiment as well (Fig. 3(d)). These results indicated that ExoQuick method can coisolate $2 \%$ free miRNA in serum regardless of the concentrations of miRNAs and RNase A not only can reduce the number of exosome-independent miRNAs, but also reduce the miRNAs in the exosomes. 
239

240

241

242

243

244

245

246

247

248

249

250

251

252

253

254

255

256

257

258

259

260

261

262

263

264

265

266

267

268

269

270

271

272

273

274

275

276

277

\section{Ultracentrifugation co-isolates almost no proportion of free miRNAs}

To investigate if UC can co-isolate free miRNAs in serum with exosomes as ExoQuick method did, higher concentration $\left(7 \times 10^{9}\right.$ copies $\left./ \mu \mathrm{L}\right)$ of cel-miR-39 was added in $500 \mu \mathrm{L}$ serum and then exosomes were extracted by UC method. The results showed that only $0.03 \%$ exogenous cel-miR-39 were co-isolated with exosomes extracted by UC method (Fig. 4(a)). Meanwhile, exosomal miR-126 and miR-152 were decreased by RNase A and miR-126 rather than miR-152 was reduced by RNase inhibitor (Fig. 4(b)). These results indicated that UC method hardly precipitate any exosome-independent miRNAs with exosomes and RNase A can reduce the miRNAs in exosomes no matter which method is used.

\section{Incubation at $37^{\circ} \mathrm{C}$ for $1 \mathrm{~h}$ reduces the amount of co-isolated free miRNAs}

In the previous experiments, we noticed that RNase inhibitor and RNase A both reduced the amount of exogenous cel-miR-39. These two reagents both require a $37{ }^{\circ} \mathrm{C}$ incubation with exosomes for $1 \mathrm{~h}$. We speculated if incubation at $37{ }^{\circ} \mathrm{C}$ for $1 \mathrm{~h}$ could cause the reduction of celmiR-39. Here, we isolated the exosomes by ExoQuick method, and the results showed that incubation at $37{ }^{\circ} \mathrm{C}$ for $1 \mathrm{~h}$ reduced the amount of cel-miR-39 as much as the RNase A did (Fig. 5(a)). Consistent with the previous results in Fig. 3(b, d) and 4(b), RNase A reduced the amount of exosomal miR-126 and miR-152, but incubation at $37{ }^{\circ} \mathrm{C}$ for $1 \mathrm{~h}$ reduced the amount of miR126 but not miR-152 like the RNase inhibitor did (Fig. 5(b)). These results indicated that incubation at $37{ }^{\circ} \mathrm{C}$ for $1 \mathrm{~h}$ without RNase inhibitor and RNase A can remove the co-isolated free miRNAs from exosome pellets, but RNase A can reduce the number of miRNAs in exosomes as well.

\section{Discussion}

Circulating miRNAs, especially exosomal miRNAs, are strongly associated with the development of different diseases, such as the manifestation, invasion and metastasis of cancers (Melo et al. 2014; Zhou et al. 2014). Therefore, circulating miRNAs can serve as novel biomarkers for diagnosis, prognosis of diseases and even drugs for diseases therapy (Cheng 2015; He et al. 2015; Huang et al. 2015). However, exosomal miRNAs are remarkably stable compared to circulating miRNAs that are not contained in exosomes, and different fractions of circulating miRNAs may play different roles in the progression of diseases, and serve as diagnosis biomarkers (Grimolizzi et al. 2017). Therefore, if miRNAs existing in exosome-free serum fractions contaminates exosomal miRNAs during laboratory studies and clinical applications, it will have a great negative impact on the sensitivity and accuracy of research and diagnostics. It has been commonly recognized that both UC and ExoQick methods co-precipitate contaminating proteins, but exosomes extracted by UC are much purer than ExoQuick on proteins (Shao et al. 2016; Tkach \& Thery 2016). However, it is still unknown if UC is also 
278 much more reliable than ExoQuick on miRNAs. Here we used exogenous cel-miR-39 as free 279 miRNA in serum and miR-126, miR-152 as exosomal miRNAs, to investigate if and to what 280 extent these two methods would co-precipitate free miRNAs in serum.

281

282

283

284

285

286

287

288

289

290

291

292

293

294

295

296

297

298

299

300

301

302

303

304

305

306

307

308

309

310

311

312

313

314

315

First, we found that compared with ExoQuick method, UC co-isolated much less exogenous cel-miR-39 with exosomes, which suggests exosomes extracted by UC are much purer than ExoQuick in exosomal miRNAs (Fig. 3(a, c) and 4(a)). Although $2 \%$ contaminating miRNAs may be too small to be considered, they can really make difference in some studies about exosomal miRNAs. Rekker et al (Rekker et al. 2014) compared UC with ExoQuick method for miRNAs profiling, and found that 17 out of 375 miRNAs had a slightly different levels between these two methods. Some of these 17 different miRNAs may be predominantly free in serum so that their levels in exosomes extracted by ExoQucik method were higher than that by UC. Based on our finding, when studying exosome-specific miRNAs, the standard UC method is preferred.

In previous studies, it was found that exosomal miRNAs could be protected from RNase by the bilayer membrane of exosomes and hence RNase A was used to degrade exosomeindependent miRNAs (Muller et al. 2014; Quackenbush et al. 2014; Rekker et al. 2014). However, our results showed that although RNase A reduced the ratio of co-precipitated miRNAs (Fig. 3(a, c) and 5(a)), it reduced the levels of exosomal miRNAs at the same time no matter which methods were used (Fig. 3(b, d), 4(b) and 5(b)). Furthermore, we have also found that a simple incubation at $37{ }^{\circ} \mathrm{C}$ for $1 \mathrm{~h}$ can reduce the amount of exosome-independent miRNAs as effectively as the addition of RNase A, but hardly reduce the amount of exosomal miRNAs at the same time (Fig. 5(a-b)).

These results suggested that merely incubation at $37{ }^{\circ} \mathrm{C}$ could remove exosome-independent miRNAs free in serum effectively, and RNase A was not recommended for degrading miRNAs out of exosomes. Earlier reports have shown that the methods of incubation at room temperature (RT) or $37{ }^{\circ} \mathrm{C}$ have been used to load drugs into exosomes (Haney et al. 2015; Ma et al. 2016). It is possible that exosomal membranes, when incubated with RNase A at $37{ }^{\circ} \mathrm{C}$ for $1 \mathrm{~h}$, could display a certain level of permeability to the enzyme, and could not fully protect miRNAs from RNase A to some degree.

It is worth noting that incubation at $37{ }^{\circ} \mathrm{C}$ for $1 \mathrm{~h}$ (with or without RNase inhibitor) slightly reduced miR-126 instead of miR-152 (Fig. 3(b, d), 4(b) and 5(b)). So, there must be a difference between miR-126 and miR-152. Turchinovich et al reported that a large part of extracellular miRNAs are associated with Ago proteins and we found from their miRNA Array data that the level of serum miR-126 associated with AGO1 and AGO2 is much higher than that of miR-152 (Turchinovich \& Burwinkel 2012; Turchinovich et al. 2011). Therefore, perhaps it is because UC and ExoQuick methods both can precipitate AGO-associated miR-126 and exosome-independent miR-126 can be degraded by incubation as cel-miR-39 did.

\section{Conclusions}


Our study provides critical information on the effect of free miRNAs in the serum when using the UC and ExoQuick methods to isolate serum exosomes and analyze exosomal miRNAs. We have found that ExoQuick method can co-precipitate much more free miRNAs in serum than UC. Furthermore, we have also found that a simple incubation at $37{ }^{\circ} \mathrm{C}$ for $1 \mathrm{~h}$ can reduce the amount of exosome-independent miRNAs as effectively as the addition of RNase A, but the latter would reduce the amount of exosomal miRNAs as well. Thus, for studies of exosomal miRNA analysis, UC method is recommended, but not the use of RNase to remove exosomeindependent miRNAs. In conclusion, these findings on the details of serum exosome isolation methods are essential for further studies on exosome-specific miRNAs.

\section{Acknowledgements}

Not applicable.

\section{References}

Alvarez ML, Khosroheidari M, Ravi RK, and DiStefano JK. 2012. Comparison of protein, microRNA, and mRNA yields using different methods of urinary exosome isolation for the discovery of kidney disease biomarkers. Kidney International 82:1024-1032.

Bartel DP. 2004. MicroRNAs: genomics, biogenesis, mechanism, and function. Cell 116:281297. 10.1016/s0092-8674(04)00045-5

Cheng G. 2015. Circulating miRNAs: roles in cancer diagnosis, prognosis and therapy. Adv Drug Deliv Rev 81:75-93. 10.1016/j.addr.2014.09.001

Colombo M, Raposo G, and Thery C. 2014. Biogenesis, secretion, and intercellular interactions of exosomes and other extracellular vesicles. Annu Rev Cell Dev Biol 30:255-289. 10.1146/annurev-cellbio-101512-122326

Grimolizzi F, Monaco F, Leoni F, Bracci M, Staffolani S, Bersaglieri C, Gaetani S, Valentino M, Amati M, Rubini C, Saccucci F, Neuzil J, Tomasetti M, and Santarelli L. 2017. Exosomal miR-126 as a circulating biomarker in non-small-cell lung cancer regulating cancer progression. Scientific Reports 7. ARTN 15277

10.1038/s41598-017-15475-6

Haney MJ, Klyachko NL, Zhaoa YL, Gupta R, Plotnikova EG, He ZJ, Patel T, Piroyan A, Sokolsky M, Kabanov AV, and Batrakova EV. 2015. Exosomes as drug delivery vehicles for Parkinson's disease therapy. Journal of Controlled Release 207:18-30. 10.1016/j.jconrel.2015.03.033

He C, Zheng S, Luo Y, and Wang B. 2018. Exosome Theranostics: Biology and Translational Medicine. Theranostics 8:237-255. 10.7150/thno.21945

He YQ, Lin JJ, Kong DL, Huang MY, Xu CK, Kim TK, Etheridge A, Luo YH, Ding YL, and Wang K. 2015. Current State of Circulating MicroRNAs as Cancer Biomarkers. Clinical Chemistry 61:1138-1155. 10.1373/clinchem.2015.241190

Huang XY, Yuan TZ, Liang MH, Du MJ, Xia S, Dittmar R, Wang D, See W, Costello BA, Quevedo F, Tan W, Nandy D, Bevan GH, Longenbach S, Sun ZF, Lu Y, Wang T, Thibodeau SN, Boardman L, Kohli M, and Wang L. 2015. Exosomal miR-1290 and miR375 as Prognostic Markers in Castration-resistant Prostate Cancer. European Urology 67:33-41. 10.1016/j.eururo.2014.07.035

Lugli G, Cohen AM, Bennett DA, Shah RC, Fields CJ, Hernandez AG, and Smalheiser NR. 2015. Plasma Exosomal miRNAs in Persons with and without Alzheimer Disease: Altered Expression and Prospects for Biomarkers. PLoS One 10. ARTN e0139233 
362

363

364

365

366

367

368

369

370

371

372

373

374

375

376

377

378

379

380

381

382

383

384

385

386

387

388

389

390

391

392

393

394

395

396

397

398

399

400

401

402

403

404

405

406

407

408

409

410

411

412

10.1371/journal.pone. 0139233

Ma JW, Zhang Y, Tang K, Zhang HF, Yin XN, Li Y, Xu PW, Sun YL, Ma RH, Ji TT, Chen JW, Zhang S, Zhang TZ, Luo SQ, Jin Y, Luo XL, Li CY, Gong HW, Long ZX, Lu JZ, Hu ZW, Cao XT, Wang N, Yang XL, and Huang B. 2016. Reversing drug resistance of soft tumor-repopulating cells by tumor cell-derived chemotherapeutic microparticles. Cell Research 26:713-727. 10.1038/cr.2016.53

Meckes DG, Jr., Shair KH, Marquitz AR, Kung CP, Edwards RH, and Raab-Traub N. 2010. Human tumor virus utilizes exosomes for intercellular communication. Proc Natl Acad Sci U S A 107:20370-20375. 10.1073/pnas.1014194107

Melo SA, Sugimoto H, O'Connell JT, Kato N, Villanueva A, Vidal A, Qiu L, Vitkin E, Perelman LT, Melo CA, Lucci A, Ivan C, Calin GA, and Kalluri R. 2014. Cancer exosomes perform cell-independent microRNA biogenesis and promote tumorigenesis. Cancer Cell 26:707721. 10.1016/j.ccell.2014.09.005

Mitchell PS, Parkin RK, Kroh EM, Fritz BR, Wyman SK, Pogosova-Agadjanyan EL, Peterson A, Noteboom J, O'Briant KC, Allen A, Lin DW, Urban N, Drescher CW, Knudsen BS, Stirewalt DL, Gentleman R, Vessella RL, Nelson PS, Martin DB, and Tewari M. 2008. Circulating microRNAs as stable blood-based markers for cancer detection. Proceedings of the National Academy of Sciences of the United States of America 105:10513-10518.

Muller L, Hong CS, Stolz DB, Watkins SC, and Whiteside TL. 2014. Isolation of biologicallyactive exosomes from human plasma. $\mathrm{J}$ Immunol Methods 411:55-65. 10.1016/j.jim.2014.06.007

Ong SG, Lee WH, Huang M, Dey D, Kodo K, Sanchez-Freire V, Gold JD, and Wu JC. 2014. Cross talk of combined gene and cell therapy in ischemic heart disease: role of exosomal microRNA transfer. Circulation 130:S60-69. 10.1161/CIRCULATIONAHA.113.007917

Pegtel DM, and Gould SJ. 2019. Exosomes. Annu Rev Biochem 88:487-514. 10.1146/annurevbiochem-013118-111902

Quackenbush JF, Cassidy PB, Pfeffer LM, Boucher KM, Hawkes JE, Pfeffer SR, Kopelovich L, and Leachman SA. 2014. Isolation of circulating microRNAs from microvesicles found in human plasma. Methods Mol Biol 1102:641-653. 10.1007/978-1-62703-727-3_34

Rekker K, Saare M, Roost AM, Kubo AL, Zarovni N, Chiesi A, Salumets A, and Peters M. 2014. Comparison of serum exosome isolation methods for microRNA profiling. Clin Biochem 47:135-138. 10.1016/j.clinbiochem.2013.10.020

Shao Y, Shen Y, Chen T, Xu F, Chen X, and Zheng S. 2016. The functions and clinical applications of tumor-derived exosomes. Oncotarget 7:60736-60751. 10.18632/oncotarget.11177

Thery C, Amigorena S, Raposo G, and Clayton A. 2006. Isolation and characterization of exosomes from cell culture supernatants and biological fluids. Curr Protoc Cell Biol Chapter 3:Unit 3 22. 10.1002/0471143030.cb0322s30

Thery C, Witwer KW, Aikawa E, Alcaraz MJ, Anderson JD, Andriantsitohaina R, Antoniou A, Arab T, Archer F, Atkin-Smith GK, Ayre DC, Bach JM, Bachurski D, Baharvand H, Balaj L, Baldacchino S, Bauer NN, Baxter AA, Bebawy M, Beckham C, Bedina Zavec A, Benmoussa A, Berardi AC, Bergese P, Bielska E, Blenkiron C, Bobis-Wozowicz S, Boilard E, Boireau W, Bongiovanni A, Borras FE, Bosch S, Boulanger CM, Breakefield $X$, Breglio AM, Brennan MA, Brigstock DR, Brisson A, Broekman ML, Bromberg JF, BrylGorecka P, Buch S, Buck AH, Burger D, Busatto S, Buschmann D, Bussolati B, Buzas El, Byrd JB, Camussi G, Carter DR, Caruso S, Chamley LW, Chang YT, Chen C, Chen $S$, Cheng L, Chin AR, Clayton A, Clerici SP, Cocks A, Cocucci E, Coffey RJ, Cordeiroda-Silva A, Couch Y, Coumans FA, Coyle B, Crescitelli R, Criado MF, D'Souza-Schorey C, Das S, Datta Chaudhuri A, de Candia P, De Santana EF, De Wever O, Del Portillo HA, Demaret T, Deville S, Devitt A, Dhondt B, Di Vizio D, Dieterich LC, Dolo V, 
413

414

415

416

417

418

419

420

421

422

423

424

425

426

427

428

429

430

431

432

433

434

435

436

437

438

439

440

441

442

443

444

445

446

447

448

449

450

451

452

453

454

455

456

457

458

459

460

461

462

463
Dominguez Rubio AP, Dominici M, Dourado MR, Driedonks TA, Duarte FV, Duncan HM, Eichenberger RM, Ekstrom K, El Andaloussi S, Elie-Caille C, Erdbrugger U, FalconPerez JM, Fatima F, Fish JE, Flores-Bellver M, Forsonits A, Frelet-Barrand A, Fricke F, Fuhrmann G, Gabrielsson S, Gamez-Valero A, Gardiner C, Gartner K, Gaudin R, Gho YS, Giebel B, Gilbert C, Gimona M, Giusti I, Goberdhan DC, Gorgens A, Gorski SM, Greening DW, Gross JC, Gualerzi A, Gupta GN, Gustafson D, Handberg A, Haraszti RA, Harrison P, Hegyesi $H$, Hendrix A, Hill AF, Hochberg FH, Hoffmann KF, Holder B, Holthofer H, Hosseinkhani B, Hu G, Huang Y, Huber V, Hunt S, Ibrahim AG, Ikezu T, Inal JM, Isin M, Ivanova A, Jackson HK, Jacobsen S, Jay SM, Jayachandran M, Jenster G, Jiang L, Johnson SM, Jones JC, Jong A, Jovanovic-Talisman T, Jung S, Kalluri R, Kano SI, Kaur S, Kawamura Y, Keller ET, Khamari D, Khomyakova E, Khvorova A, Kierulf P, Kim KP, Kislinger T, Klingeborn M, Klinke DJ, 2nd, Kornek M, Kosanovic MM, Kovacs AF, Kramer-Albers EM, Krasemann S, Krause M, Kurochkin IV, Kusuma GD, Kuypers S, Laitinen S, Langevin SM, Languino LR, Lannigan J, Lasser C, Laurent LC, Lavieu G, Lazaro-Ibanez E, Le Lay S, Lee MS, Lee YXF, Lemos DS, Lenassi M, Leszczynska A, Li IT, Liao K, Libregts SF, Ligeti E, Lim R, Lim SK, Line A, Linnemannstons K, Llorente A, Lombard CA, Lorenowicz MJ, Lorincz AM, Lotvall J, Lovett J, Lowry MC, Loyer X, Lu Q, Lukomska B, Lunavat TR, Maas SL, Malhi H, Marcilla A, Mariani J, Mariscal J, Martens-Uzunova ES, Martin-Jaular L, Martinez MC, Martins VR, Mathieu M, Mathivanan S, Maugeri M, McGinnis LK, McVey MJ, Meckes DG, Jr., Meehan KL, Mertens I, Minciacchi VR, Moller A, Moller Jorgensen M, MoralesKastresana A, Morhayim J, Mullier F, Muraca M, Musante L, Mussack V, Muth DC, Myburgh KH, Najrana T, Nawaz M, Nazarenko I, Nejsum P, Neri C, Neri T, Nieuwland R, Nimrichter L, Nolan JP, Nolte-'t Hoen EN, Noren Hooten N, O'Driscoll L, O'Grady T, O'Loghlen A, Ochiya T, Olivier M, Ortiz A, Ortiz LA, Osteikoetxea X, Ostergaard O, Ostrowski M, Park J, Pegtel DM, Peinado H, Perut F, Pfaffl MW, Phinney DG, Pieters BC, Pink RC, Pisetsky DS, Pogge von Strandmann E, Polakovicova I, Poon IK, Powell BH, Prada I, Pulliam L, Quesenberry P, Radeghieri A, Raffai RL, Raimondo S, Rak J, Ramirez MI, Raposo G, Rayyan MS, Regev-Rudzki N, Ricklefs FL, Robbins PD, Roberts DD, Rodrigues SC, Rohde E, Rome S, Rouschop KM, Rughetti A, Russell AE, Saa P, Sahoo S, Salas-Huenuleo E, Sanchez C, Saugstad JA, Saul MJ, Schiffelers RM, Schneider R, Schoyen TH, Scott A, Shahaj E, Sharma S, Shatnyeva O, Shekari F, Shelke GV, Shetty AK, Shiba K, Siljander PR, Silva AM, Skowronek A, Snyder OL, 2nd, Soares RP, Sodar BW, Soekmadji C, Sotillo J, Stahl PD, Stoorvogel W, Stott SL, Strasser EF, Swift S, Tahara H, Tewari M, Timms K, Tiwari S, Tixeira R, Tkach M, Toh WS, Tomasini R, Torrecilhas AC, Tosar JP, Toxavidis V, Urbanelli L, Vader P, van Balkom BW, van der Grein SG, Van Deun J, van Herwijnen MJ, Van Keuren-Jensen K, van Niel G, van Royen ME, van Wijnen AJ, Vasconcelos MH, Vechetti IJ, Jr., Veit TD, Vella LJ, Velot E, Verweij FJ, Vestad B, Vinas JL, Visnovitz T, Vukman KV, Wahlgren J, Watson DC, Wauben MH, Weaver A, Webber JP, Weber V, Wehman AM, Weiss DJ, Welsh JA, Wendt S, Wheelock AM, Wiener Z, Witte L, Wolfram J, Xagorari A, Xander P, Xu J, Yan X, Yanez-Mo M, Yin H, Yuana Y, Zappulli V, Zarubova J, Zekas V, Zhang JY, Zhao Z, Zheng L, Zheutlin AR, Zickler AM, Zimmermann P, Zivkovic AM, Zocco D, and Zuba-Surma EK. 2018. Minimal information for studies of extracellular vesicles 2018 (MISEV2018): a position statement of the International Society for Extracellular Vesicles and update of the MISEV2014 guidelines. J Extracell Vesicles 7:1535750.

10.1080/20013078.2018.1535750

Thery C, Zitvogel L, and Amigorena S. 2002. Exosomes: composition, biogenesis and function. Nat Rev Immunol 2:569-579. 10.1038/nri855

Tkach M, and Thery C. 2016. Communication by Extracellular Vesicles: Where We Are and Where We Need to Go. Cell 164:1226-1232. 10.1016/j.cell.2016.01.043 
464

465

466

467

468

469

470

471

472

473

474

475

476

477

478

479

480

481
Turchinovich A, and Burwinkel B. 2012. Distinct AGO1 and AGO2 associated miRNA profiles in human cells and blood plasma. Rna Biology 9:1066-1075. 10.4161/rna.21083

Turchinovich A, Weiz L, Langheinz A, and Burwinkel B. 2011. Characterization of extracellular circulating microRNA. Nucleic Acids Res 39:7223-7233. 10.1093/nar/gkr254

Umezu T, Ohyashiki K, Kuroda M, and Ohyashiki JH. 2013. Leukemia cell to endothelial cell communication via exosomal miRNAs. Oncogene 32:2747-2755.

Valadi H, Ekstrom K, Bossios A, Sjostrand M, Lee JJ, and Lotvall JO. 2007. Exosome-mediated transfer of mRNAs and microRNAs is a novel mechanism of genetic exchange between cells. Nature Cell Biology 9:654-U672. 10.1038/ncb1596

Zhang J, Ma J, Long K, Qiu W, Wang Y, Hu Z, Liu C, Luo Y, Jiang A, Jin L, Tang Q, Wang X, Li $X$, and Li M. 2017. Overexpression of Exosomal Cardioprotective miRNAs Mitigates Hypoxia-Induced H9c2 Cells Apoptosis. Int J Mol Sci 18. 10.3390/ijms18040711

Zhou W, Fong MY, Min Y, Somlo G, Liu L, Palomares MR, Yu Y, Chow A, O'Connor ST, Chin AR, Yen Y, Wang Y, Marcusson EG, Chu P, Wu J, Wu X, Li AX, Li Z, Gao H, Ren X, Boldin MP, Lin PC, and Wang SE. 2014. Cancer-secreted miR-105 destroys vascular endothelial barriers to promote metastasis. Cancer Cell 25:501-515.

10.1016/j.ccr.2014.03.007 
Figure 1

Figure 1. The experimental flowchart of this research.

(I) exogenously added cel-miR-39 in serum, blank control: $\mathrm{ddH}_{2} \mathrm{O}$, (II) isolation of exosomes by ExoQuick or UC method, (III) using $10 \mu \mathrm{g} / \mathrm{mL}, 20 \mu \mathrm{g} / \mathrm{mL}, 30 \mu \mathrm{g} / \mathrm{mL}$ RNase A or $1 \mathrm{U} / \mu \mathrm{L}$ RNase inhibitor or nothing with incubation at $37^{\circ} \mathrm{C}$ for $1 \mathrm{~h}$ or nothing (control) to treat exosome pellets, (IV) extraction of total RNA including miRNA by miRNeasy Mini Kit, (V) detecting the quantity of cel-miR-39, miR-126 or miR-152 in exosome samples by qRT-PCR. 
Exosome Isolation

Treatment

RNA

Extraction

qRT-PCR

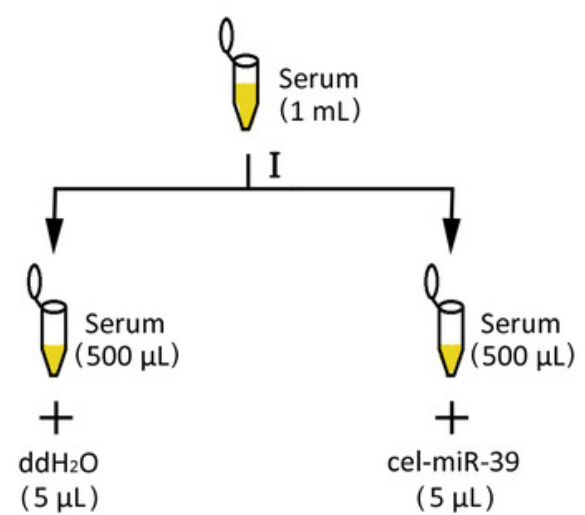

II

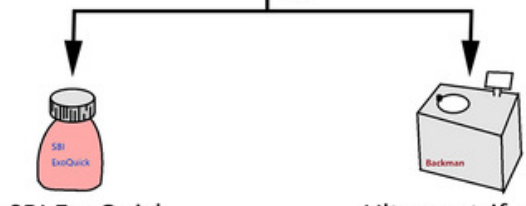

SBI ExoQuick

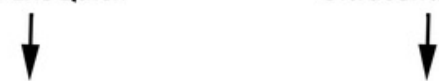

$b$

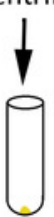

$+$

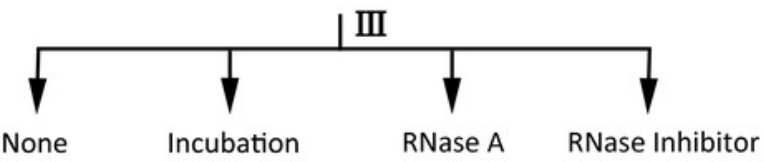

IIV

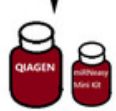

QIAGEN miRNeasy Mini Kit

I

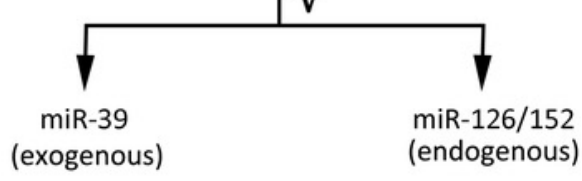




\section{Figure 2}

Figure 2. The characterization of exosomes isolated from human serum.

(a, d) The morphology of exosomes. Scale bars: $200 \mathrm{~nm}$. (b, d) The particle size distribution of exosomes. (e, f) The level of exosome-associated protein, CD63, TSG101 and CD9.

a

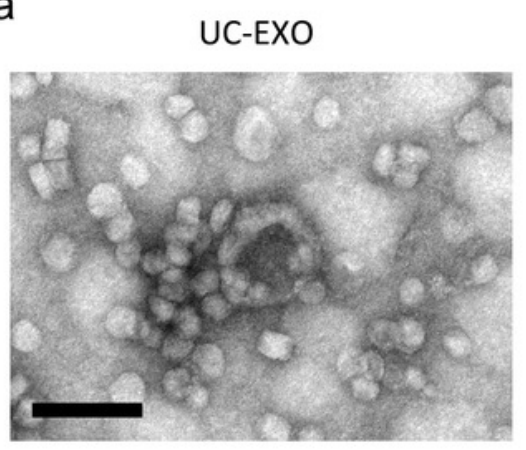

d

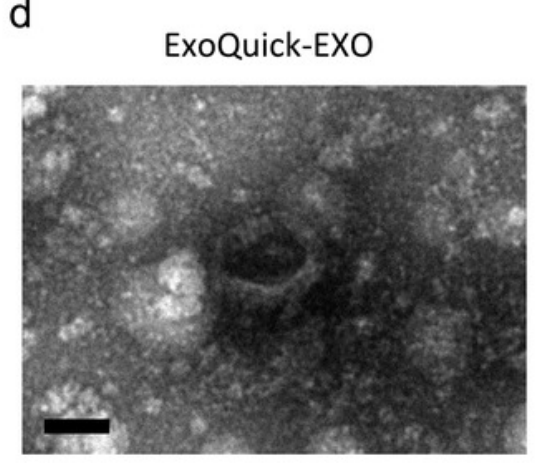

b

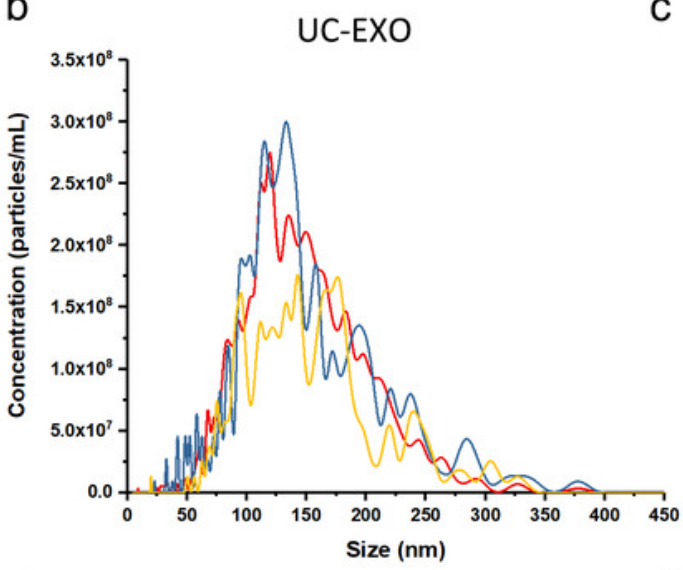

e

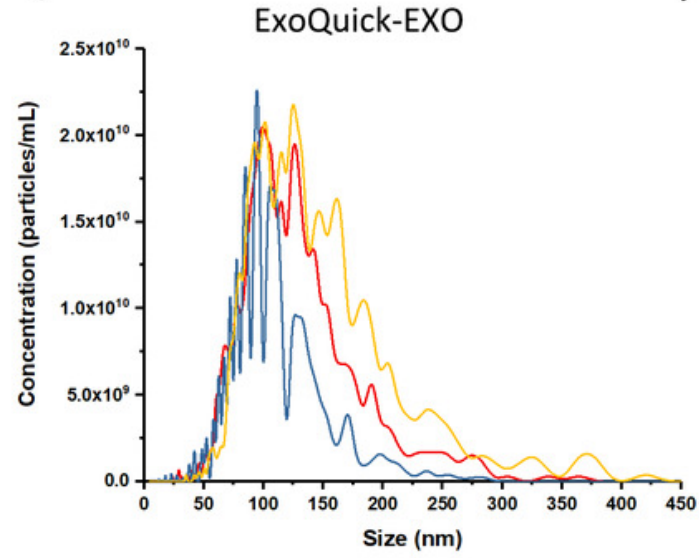

C

UC-EXO

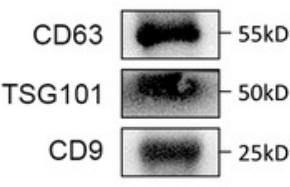

ExoQuick-EXO

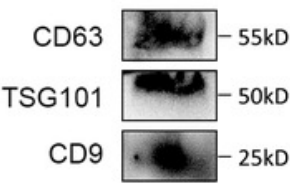




\section{Figure 3}

Figure 3. ExoQuick method co-isolates a small proportion of free miRNAs.

(a-b) High concentration $\left(7 \times 10^{9}\right.$ copies $\left./ \mu \mathrm{L}\right)$ of cel-miR-39 were added in serum. Exosomes were extracted by ExoQuick method. (a) The ratio of co-precipitated cel-miR-39 in different samples to standard sample $\left(10^{9}\right.$ copies/ $/ \mathrm{L}$ cel-miR-39). ${ }^{* * *} p<0.001 \mathrm{vs.}$. Control. (b) The amount of exosomal miR-126 and miR-152 in different samples. ${ }^{* *} p<0.01,{ }^{* * *} p<0.001$ vs. Control. ${ }^{*} p<0.05,{ }^{*}{ }^{*} p<0.01$ vs. RNase Inhibitor. (c-d) Low concentration $\left(7 \times 10^{6}\right.$ copies $\left./ \mu L\right)$ of cel-miR-39 were added in serum. Exosomes were extracted by ExoQuick method. (c) The ratio of co-precipitated cel-miR-39 in different samples to standard sample $\left(10^{6} \mathrm{copies} / \mu \mathrm{L}\right.$ celmiR-39). (d) The amount of exosomal miR-126 and miR-152 in different samples. ${ }^{* * *} p<0.001$ vs. Control. ${ }^{\# \#} p<0.001$ vs. RNase Inhibitor. 
a

The ratio of cel-miR-39 copies (sample/standard)

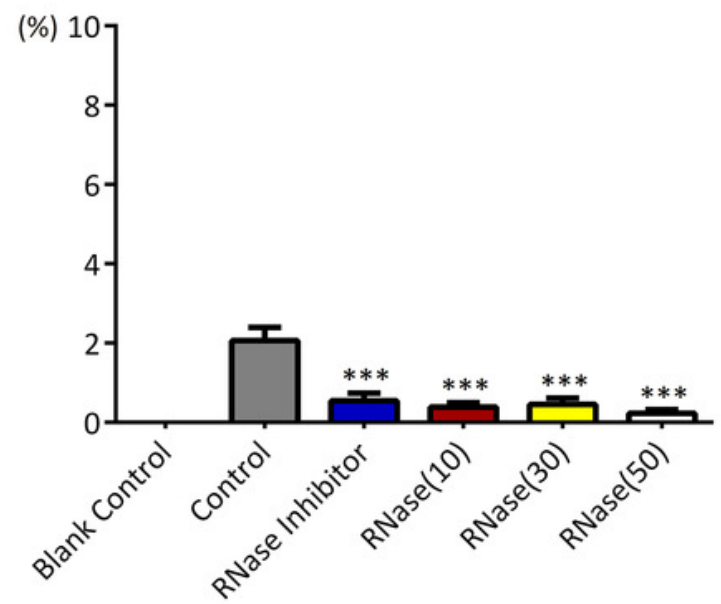

C

The ratio of cel-miR-39 copies (sample/standard)

(\%)

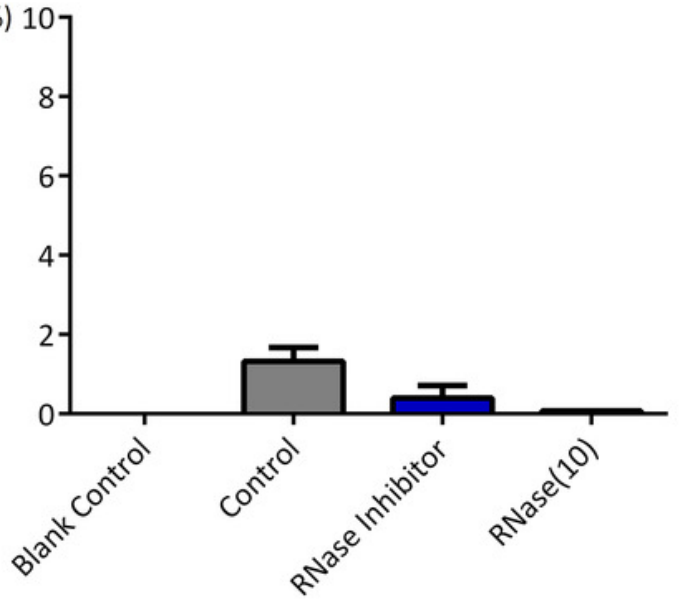

b

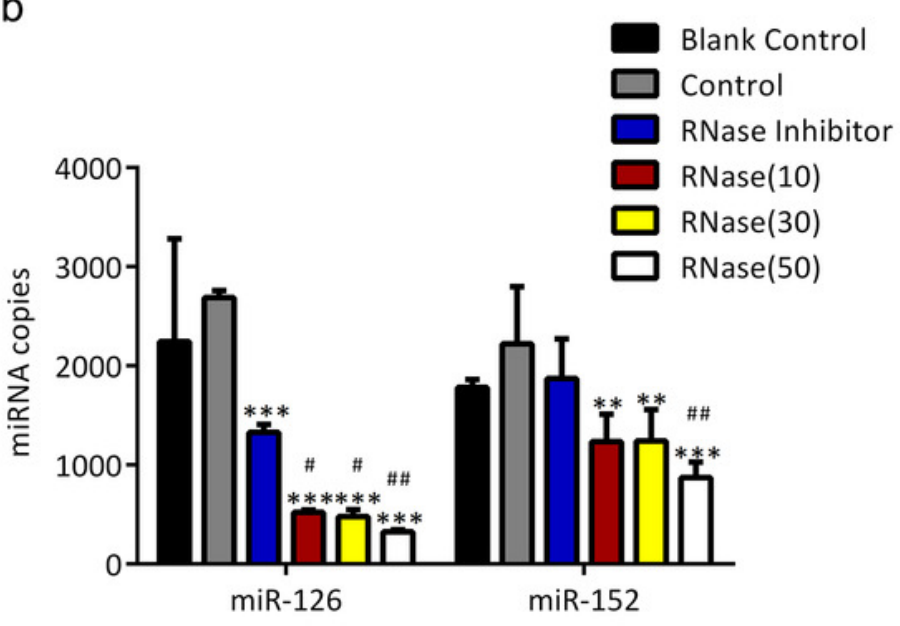

d

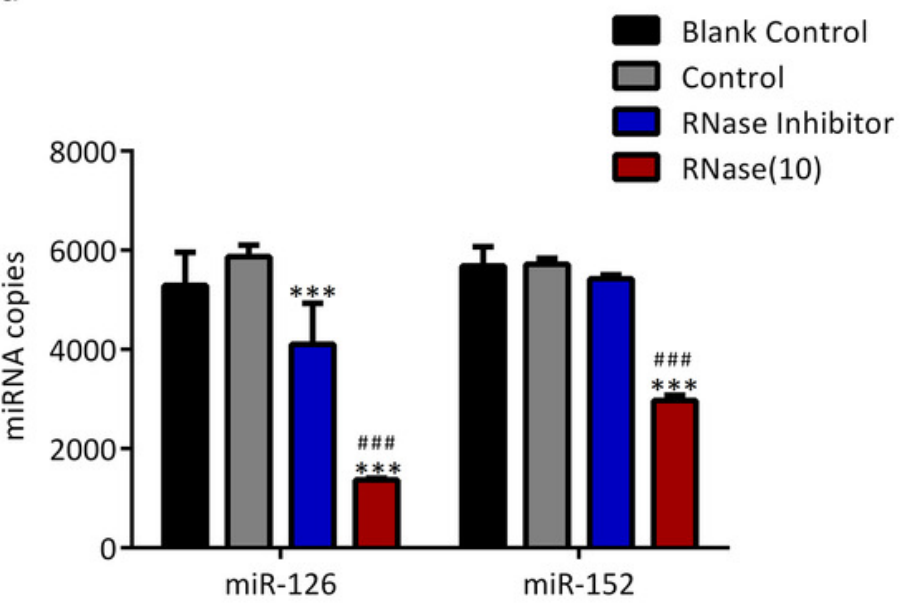




\section{Figure 4}

Figure 4. Ultracentrifugation (UC) method co-isolates almost no proportion of free miRNAs.

(a-b) High concentration $\left(7 \times 10^{9}\right.$ copies $\left./ \mu \mathrm{L}\right)$ of cel-miR-39 were added in serum. Exosomes were extracted by UC method. (a) The ratio of co-precipitated cel-miR-39 in different samples to standard sample ( $10^{9}$ copies/ $\mu \mathrm{L}$ cel-miR-39). (b) The amount of exosomal miR-126 and miR-152 in different samples. ${ }^{* *} p<0.01,{ }^{* * *} p<0.001$ vs. Control. ${ }^{*} p<0.05$ vs. RNase Inhibitor.

a

The ratio of cel-miR-39 copies (sample/standard)

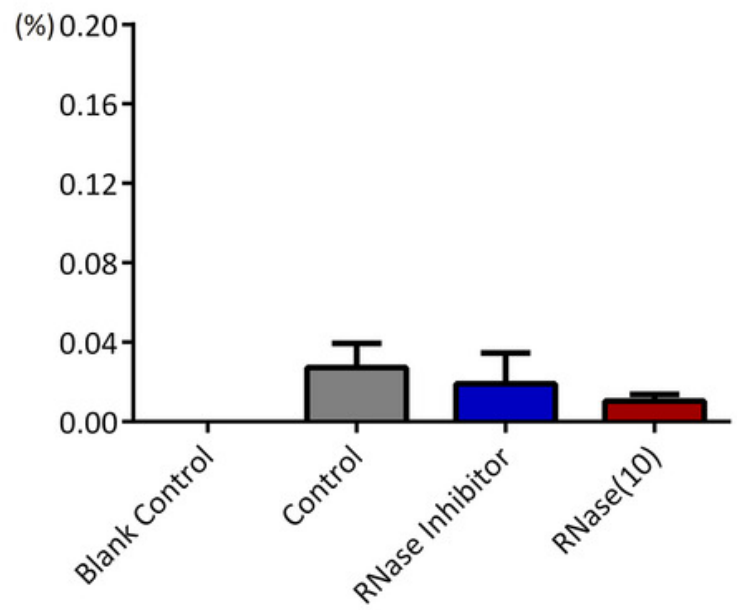

b

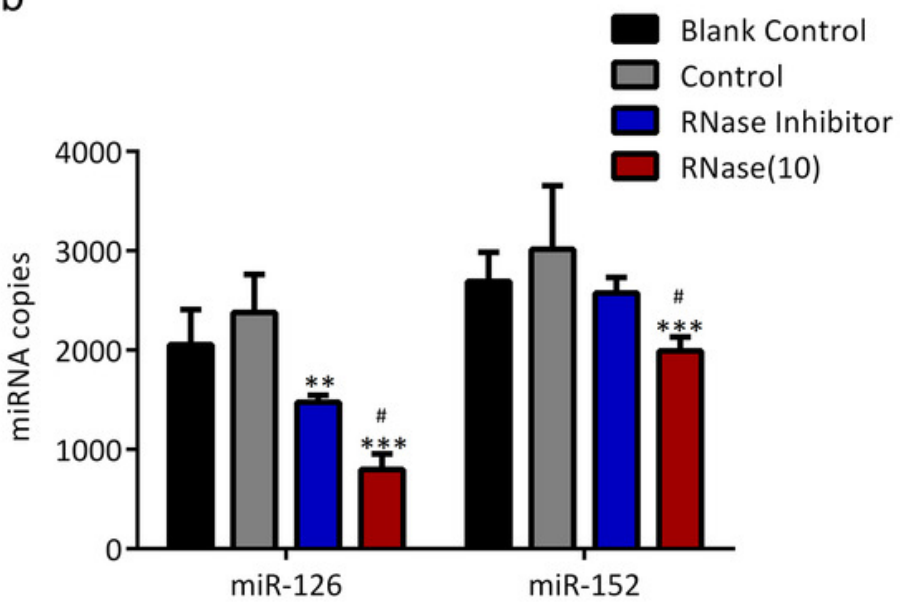




\section{Figure 5}

Figure 5. Incubation at $37^{\circ} \mathrm{C}$ for $1 \mathrm{~h}$ reduces the amount of co-isolated free miRNAs.

(a-b) High concentration $\left(7 \times 10^{9}\right.$ copies $\left./ \mu \mathrm{L}\right)$ of cel-miR-39 were added in serum. Exosomes were extracted by ExoQuick method. (a) The ratio of co-precipitated cel-miR-39 in different samples to standard sample $\left(10^{9}\right.$ copies $/ \mu \mathrm{L}$ cel-miR-39). ${ }^{*} p<0.001$ vs. Control. (b) The amount of exosomal miR-126 and miR-152 in different samples. ***p<0.001 vs. Control. \# \# ${ }^{*} p<0.05$ vs. Incubation.

a

The ratio of cel-miR-39 copies (sample/standard)

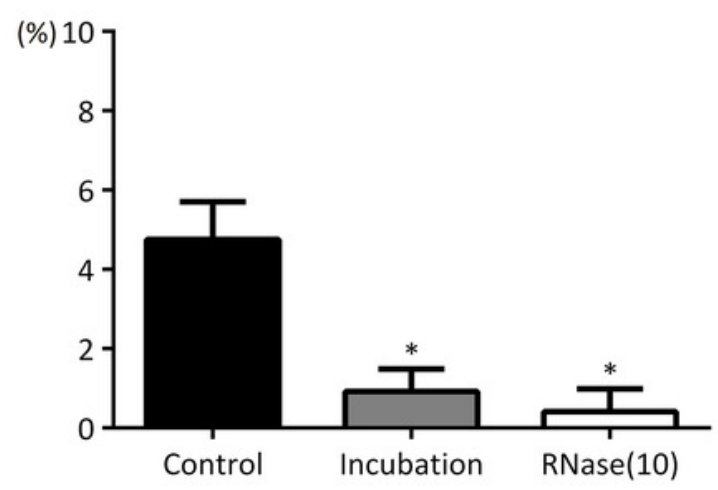

b

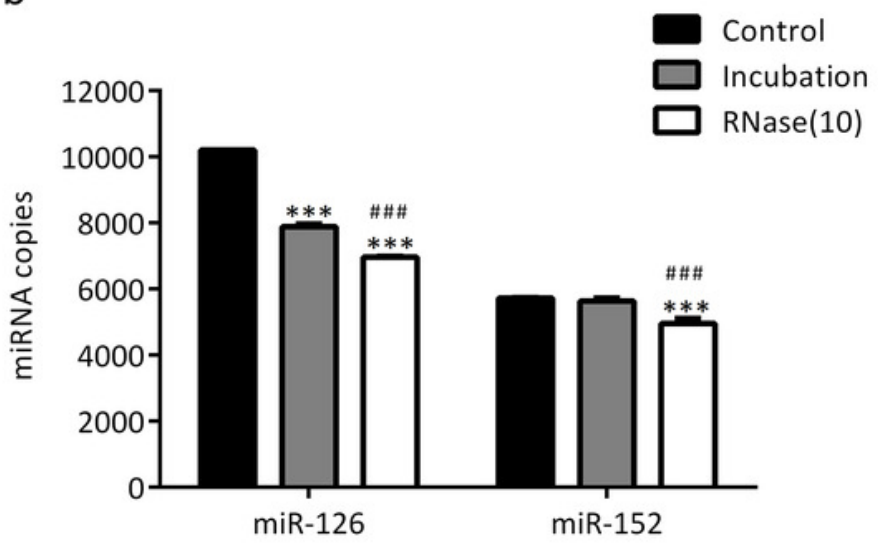

